\title{
Consecutive Successful Pregnancies after Conservative Manage- ment of Early Stage Endometrial Carcinoma
}

\author{
Chia-Chi Yen ${ }^{1}$, Chieh-Yi Kang ${ }^{1}$, Hsing-Chun Tsai ${ }^{1}$, Ming-Ting Chung ${ }^{1}$, Meng-Chen Tsai $^{2}$ and Yung- \\ Chieh Tsai ${ }^{1,3,4^{*}}$ \\ ${ }^{1}$ Department of Obstetrics and Gynecology, Chi-Mei Medical Center, Tainan, Taiwan \\ ${ }^{2}$ Pathology Center, Chi-Mei Medical Center, Tainan, Taiwan \\ ${ }^{3}$ Department of Sports Management, Chia Nan University of Pharmacy and Science, Tainan, Taiwan \\ ${ }^{4}$ Department of Medicine, Taipei Medical University, Taipei, Taiwan
}

*Corresponding author: Yung-Chieh Tsai, Center for Reproductive Medicine, Department of Obstetrics and Gynecology, Chi Mei Medical Center, 901 Chung Hwa Road, Yung-Kang City, Tainan 71010, Taiwan

\begin{abstract}
Endometrial cancer is one of the most frequent gynecological cancers in women. According to a recent national survey, endometrial cancer has increased rapidly in the past 30 years in Taiwan and the incidence of endometrioid adenocarcinoma has increased more and more quickly in younger age. Although total hysterectomy is usually recommended to eradicate the malignant lesion, this procedure may not be suitable for a young nulliparous woman, especially when the endometrial cancer is early stage. We report a case of consecutive successful pregnancies by in vitro fertilization and embryo transfer (IVF-ET) after conservative management of early stage endometrial carcinoma. To our knowledge, this is the first such case ever been reported in Taiwan. We believe this case will be very encouraging to younger women with early stage endometrial cancer.
\end{abstract}

\section{Keywords}

Endometrial cancer, Fertility-sparing therapy, Progestin

\section{Introduction}

Endometrial cancer is one of the most frequent gynecological cancers in women. While the majority of cases are diagnosed in post-menopausal women, up to $14 \%$ of cases happen in the pre-menopausal women including $4 \%$ diagnosed in women less than 40 years of age $[1,2]$. According to a recent national survey in Taiwan, cases of hormone- dependent type I endometrial cancer have increased rapidly in the past 30 years and the incidence of endometrioid adenocarcinoma has increased more and more quickly in younger age [3]. Standard management of endometrial cancer at diagnosis involves hysterectomy with bilateral salpingo-oophorectomy, followed by chemotherapy with or without radiation therapy [4]. Since some of the young endometrial cancer patients may not have completed their family planning at the time of diagnosis, fertility preservation has become one of the most important issues in the past few years. Here we present the case of a patient who achieved consecutive successful pregnancies via assisted reproduction technologies after conservative management of early stage endometrial carcinoma.

\section{Case Description}

The patient, a 39-year-old woman, BMI 26.6, came to our hospital due to primary infertility for the past seven years. She had an irregular menstruation and basal body temperature revealed no biphasic change. Multiple small follicles in both ovaries with necklace like appearance were noted during ultrasound examination. The serum level of follicular stimulating hormone (FSH) was $5.28 \mathrm{mlU} / \mathrm{mL}$ (reference range: 3.03-8.08 $\mathrm{mIU} / \mathrm{mL}$ ) and luteinizing hormone (LH) was $9.6 \mathrm{mIU} /$ $\mathrm{mL}$ (reference range: $1.80-11.78 \mathrm{mIU} / \mathrm{mL}$ ). Diagnosis of polycystic ovary syndrome was made according to Rotterdam criteria (Rotterdam ESHRE/ASRM-Sponsored PCOS consensus workshop group, 2004) [5]. The patient did not return after one cycle of unsuccessful ar- 

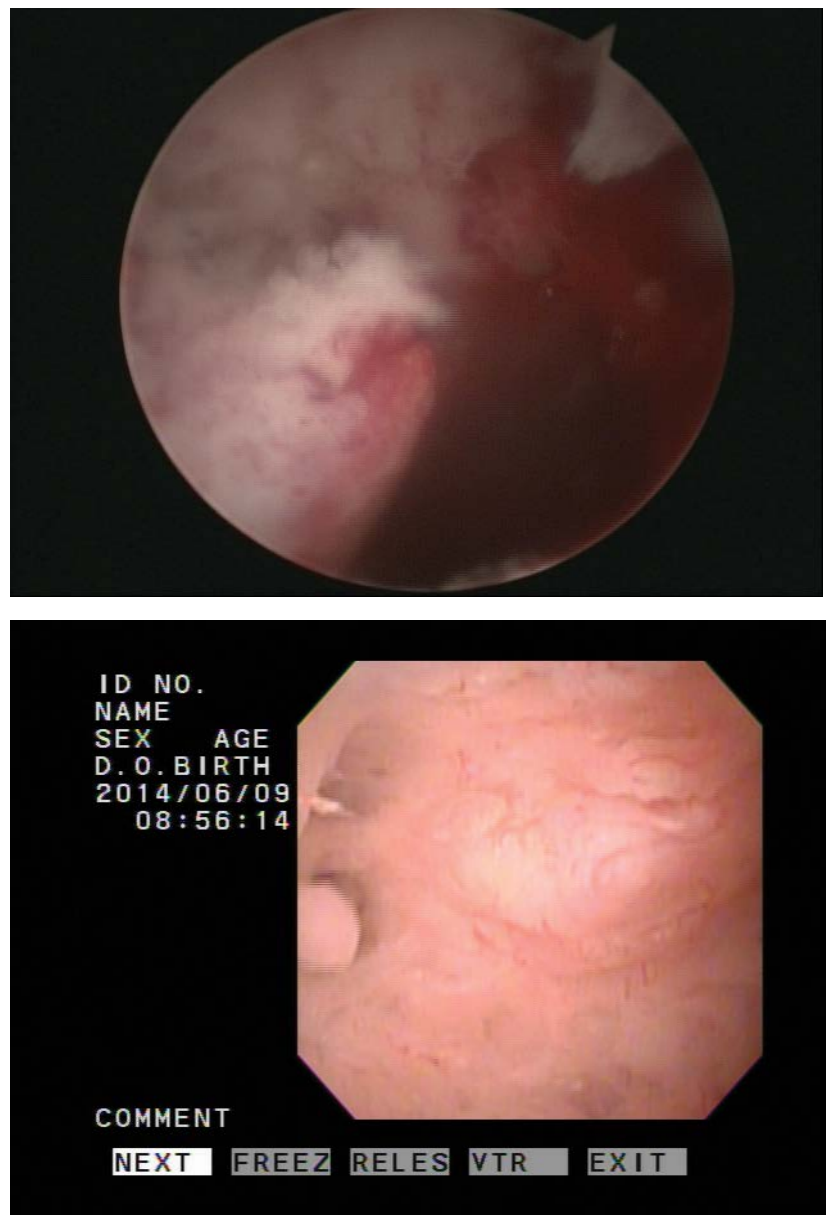

Figure 1: Hysteroscopic finding of the endometrial cancer.

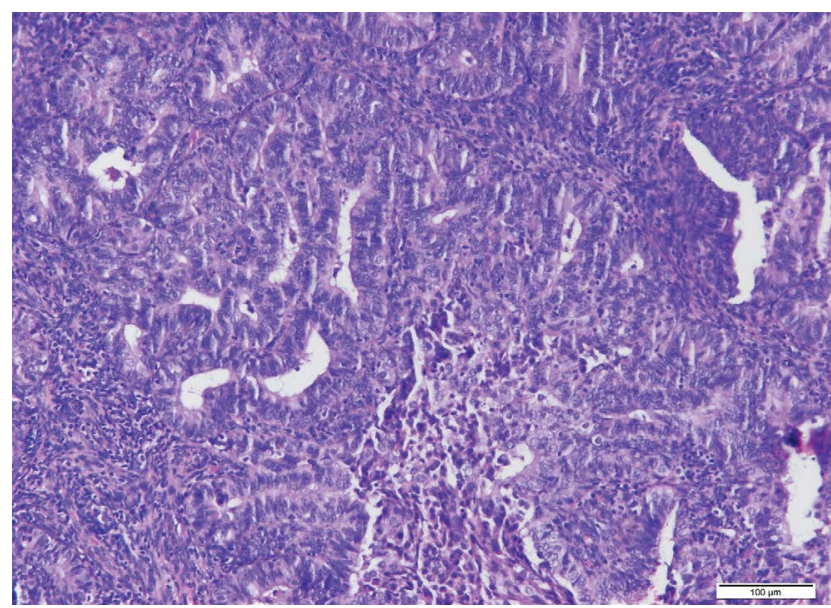

Figure 2: Pre-therapy. Endometrial glands with neoplastic transformation, H\&E, 200x.

tificial insemination. She visited our hospital again two years later requesting in vitro fertilization and embryo transfer (IVF-ET) directly due to advanced maternal age. Routine hysteroscopy (HSC) examination before treatment found a small vessel-rich mass in the uterine cavity (Figure 1). Biopsy was performed and endometrioid adenocarcinoma was diagnosed (Figure 2). Magnetic resonance imaging (MRI) of the uterus showed an intact endomyometrial junctional zone. There was no pathology regarding lymph nodes or metastatic disease nor suspicious adnexal mass (Figure 3 ).

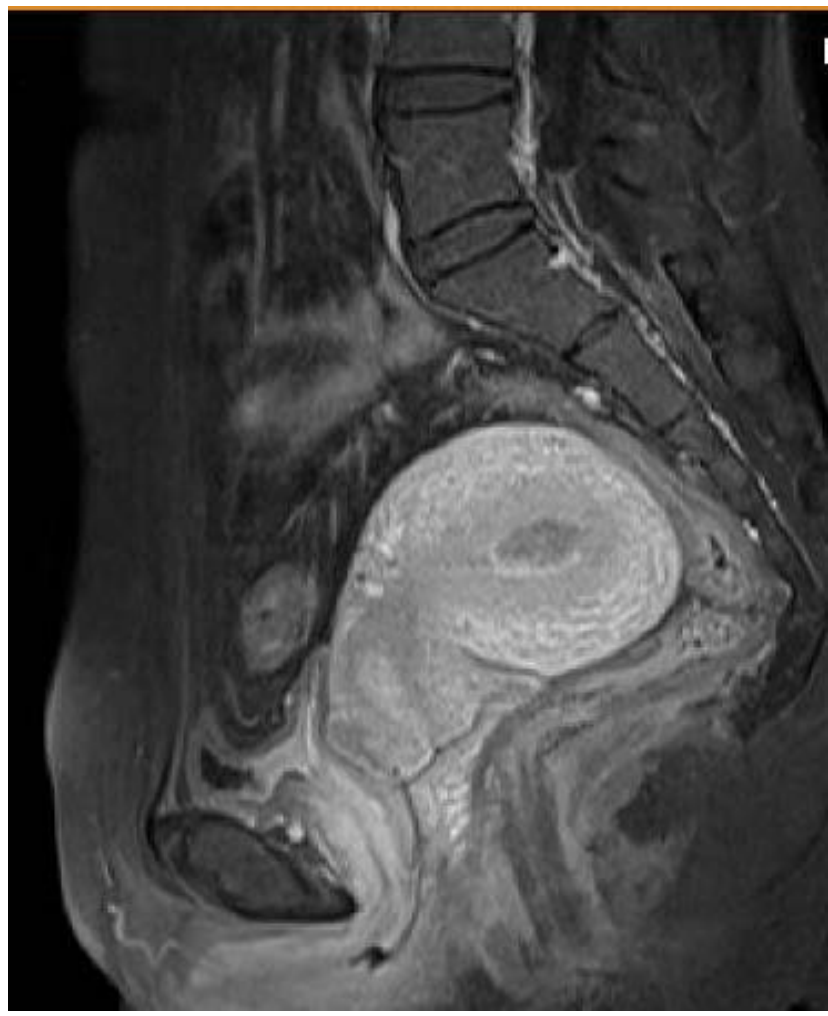

Figure 3: MRI finding before Fertility-sparing therapy.

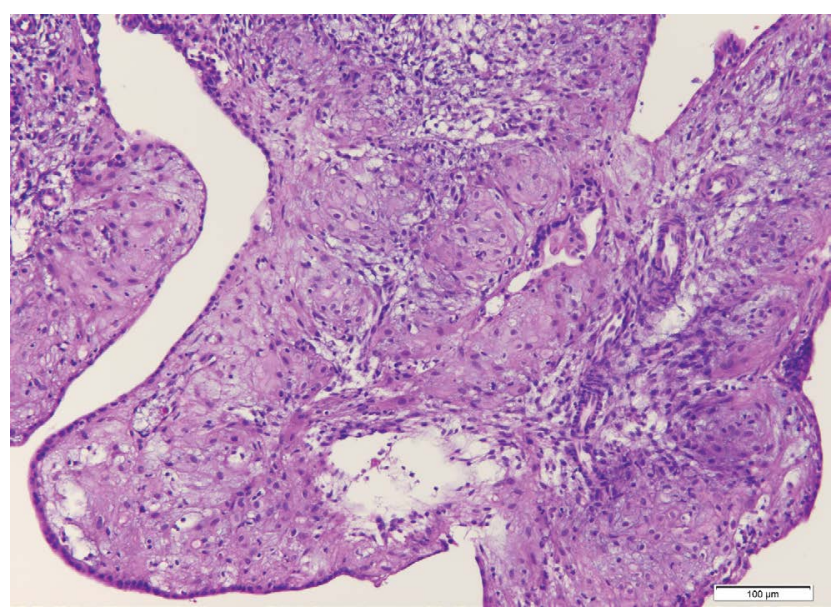

Figure 4: Post-therapy with high-dose systemic progesterone. Stroma with decidual transformation (drug-induced). Hyperplasia within the glandular tubes and on the endometrium surface - foci of residual hyperplasia, H\&E, 200x.

Due to the patient's desire to retain her child-bearing potential, conservative management was decided after thorough consultation. Dilation and curettage (D\&C) was arranged and the report showed low grade endometrioid adenocarcinoma without evidence of lymphvascular space invasion (LVSI). There were no contraindications to medical therapy nor pregnancy. Ovulation induction followed by IVF was performed and all 4 embryos were frozen. Medroxyprogesterone acetate (MPA) $5 \mathrm{mg}$ twice daily was given after the D\&C for 24 days until oocyte retrieval. She then received medroxyprogesterone acetate (Farlutal) at a dose of $250 \mathrm{mg} / \mathrm{d}$ for four months. A repeat HSC with endometrial D\&C revealed no residual cancer (Figure 4). Frozen embryo 
transfer (FET) with three embryos was arranged but was unsuccessful. Another IVF-ET was arranged immediately during the next cycle. Three embryos were transferred, and three embryos were frozen. A single gestational sac was noted at transvaginal ultrasonography ten days after the positive pregnancy test. The course of pregnancy was uneventfully, and she gave birth to a healthy female baby at term. Hysterectomy was recommended for her safety, but patient refused and requested FET during the following year. Farlutal at a dose of $250 \mathrm{mg} / \mathrm{d}$ was prescribed and FET with the remaining three embryos was performed after normal endometrial biopsy. She gave birth to her second female child nine months after the second FET. She is currently receiving regular follow up at our hospital and preparing for hysterectomy.

Progestin treatment of atypical hyperplasia and well differentiated carcinoma of the endometrium in women under age of 40 was first published by Randall, et al. at 1997 [6]. Since then, many patients with early stage endometrial carcinoma have been reported to have achieved successful pregnancies after conservative medical management [7-9]. Because recurrence after successful fertility-sparing therapy of endometrial cancer is very high, prophylactic hysterectomy is usually performed once patient has given birth to a child. Until now, there are no data regarding how many pregnancies a woman could support after fertility-sparing therapy of early endometrial cancer. The case we present here with two consecutive successful pregnancies is encouraging for women diagnosed with early stage endometrial carcinoma who wish to have more than one child.

According to the recommendation from American College of Gynecology (ACOG) in 2011, for women with low risk of endometrial cancer (grade 1 or 2, stage IA, endometrioid endometrial carcinoma, confined to the endometrium) who wish to preserve their fertility, conservative management such as progestin-based therapy can be considered as long as she fulfilled the following criteria; Absence of suspicious or metastatic disease on imaging, absence of lymphovascular space invasion (LVSI) on adequate curettage specimen, no evidence of a suspicious adnexal mass on CT or pelvic ultrasound imaging, strong and diffuse expression of progesterone receptors on immunohistochemical staining of an endometrial biopsy or curettage specimen, no contraindications to medical therapy or pregnancy were all basic requirements to perform this "fertility sparing therapy" [10-12].

Current fertility-sparing treatment modalities mainly comprise hormonal therapies involving progestins, progestin-releasing intrauterine devices, natural progesterone, oral contraceptives, selective estrogen receptor modulators, gonadotropin-releasing hormone agonist, and aromatase inhibitors. Among which, oral progestin therapy is the one of the most commonly used with well-established efficacy. Progesterone has long been known to suppress the growth of endometrial cancer by down-regulating estrogen receptors, activating enzymes in estrogen metabolism, involving cell cycle regulation by cyclin-dependent kinase (Cdk) [13], and reinforcing p27 (a cyclin E-Cdk2 complex inhibitor) expression resulting in suppression of the cell cycle [14]. Treatment of low-risk endometrial cancer with oral MPA in a range of doses from 2.5 to $1500 \mathrm{mg}$ daily has been reported. The most commonly used dose is $600 \mathrm{mg}$ daily. The range of doses of megestrol acetate (MA) has been reported from 10 to $400 \mathrm{mg}$ daily and the most commonly used dose is $160 \mathrm{mg}$ daily [15]. The choice of dose is based on a need to balance efficacy with toxicity. For patients who dislikes oral medicine, progestin-releasing intrauterine devices seem to be an optimal alternative. Wei J [16] conducted a meta-analysis comparing the efficacy of different fertility-sparing treatments in patient with early endometrial cancer or atypical complex hyperplasia. Complete remission (CR) was found in $71 \%$ of patients managed with progestin, $76 \%$ with progestin-releasing intrauterine devices and $87 \%$ for women treated with both. However, the pregnancy rate was $34 \%$ in progestin group (live birth rate of $20 \%$ ), $18 \%$ in IUD group (live birth rate of $14 \%$ ), and $40 \%$ in progestin plus IUD group (live birth rate of $35 \%$ ). They concluded for patients with early endometrial cancer and atypical complex hyperplasia that treatments with progestin, with or without IUD or IUD alone, can achieve good CR rate; however, the pregnancy outcomes might be less in patients treated with IUD alone.

Though fertility-sparing treatments seem promising, these are not without risk. For example, the optimal dosage and duration of oral progestin remains to be determined and there is a high rate of recurrence after fertility-sparing therapy [17]. Recurrence risk ranges from 24 to 41 percent, with a mean time to recurrence between 15 and 35 months $[18,19]$. Therefore, thorough selection of patients through comprehensive pretreatment evaluation and counseling is needed before offering this nonstandard therapy. Close follow-up during therapy is very important and, hysterectomy after completion of childbirth is recommended as a definitive treatment. Our patient who achieved two consecutive successful pregnancies after diagnosis of early endometrial cancer is encouraging and demonstrates the feasibility of fertility sparing therapy.

\section{References}

1. Duska LR, Garrett A, Rueda BR, Haas J, Chang $Y$, et al. (2001) Endometrial cancer in women 40 years old or younger. Gynecol Oncol 83: 388-393.

2. Gitsch G, Hanzal E, Jensen D, Hacker NF (1995) Endometrial cancer in premenopausal women 45 years and younger. Obstet Gynecol 85: 504-508.

3. Huang CY, Chen CA, Chen YL, Chiang CJ, Hsu TH, et al. (2012) Nationwide surveillance in uterine cancer: Survival analysis and the importance of birth cohort: 30 -year population-based registry in Taiwan. PLoS One 7: e51372. 
4. de Haydu C, Black JD, Schwab CL, English DP, Santin AD (2016) An update on the current pharmacotherapy for endometrial cancer. Expert Opin Pharmacother 17: 489-499.

5. Rotterdam ESHRE/ASRM-Sponsored PCOS consensus workshop group (2004) Revised 2003 consensus on diagnostic criteria and long-term health risks related to polycystic ovary syndrome (PCOS). Hum Reprod 19: 41-47.

6. Randall TC, Kurman RJ (1997) Progestin treatment of atypical hyperplasia and well-differentiated carcinoma of the endometrium in women under age 40. Obstet Gynecol 90: 434-440.

7. Chao AS, Chao A, Wang CJ, Lai CH, Wang HS (2011) Obstetric outcomes of pregnancy after conservative treatment of endometrial cancer: Case series and literature review. Taiwan J Obstet Gynecol 50: 62-66.

8. Pashov Al, Tskhay VB, lonouchene SV (2012) The combined $\mathrm{GnRH}$-agonist and intrauterine levonorgestrel-releasing system treatment of complicated atypical hyperplasia and endometrial cancer: A pilot study. Gynecol Endocrinol 28: 559-561.

9. Magnowska M, Nowak-Markwitz E, Frankowski A, Rzeszutko W, Spaczyński M (2015) Successful pregnancy after conservative management of early stage endometrial carcinoma in a young nulliparous woman. Arch Med Sci 11: 237-239.

10. Gunderson CC, Fader AN, Carson KA, Bristow RE (2012) Oncologic and reproductive outcomes with progestin therapy in women with endometrial hyperplasia and grade 1 adenocarcinoma: A systematic review. Gynecol Oncol 125: 477-482.

11. Baker J, Obermair A, Gebski V, Janda M (2012) Efficacy of oral or intrauterine device-delivered progestin in patients with complex endometrial hyperplasia with atypia or early endometrial adenocarcinoma: A meta-analysis and systematic review of the literature. Gynecol Oncol 125: 263-270.
12. Gracia CR, Jeruss JS (2013) Lives in the balance: women with cancer and the right to fertility care. J Clin Oncol 31 : 668-669.

13. Banno K, Kisu I, Yanokura M, Tsuji K, Masuda K, et al. (2012) Progestin therapy for endometrial cancer: The potential of fourth-generation progestin (review). Int J Oncol 40: 1755-1762.

14. Shimizu Y, Takeuchi T, Mita S, Mizuguchi K, Kiyono T, et al. (2009) Dienogest, a synthetic progestin, inhibits the proliferation of immortalized human endometrial epithelial cells with suppression of cyclin D1 gene expression. Mol Hum Reprod 15: 693-701.

15. Park JY, Kim DY, Kim JH, Kim YM, Kim KR, et al. (2013) Long-term oncologic outcomes after fertility-sparing management using oral progestin for young women with endometrial cancer (KGOG 2002). Eur J Cancer 49: 868-874.

16. Wei J, Zhang W, Feng L, Gao W (2017) Comparison of fertility-sparing treatments in patients with early endometrial cancer and atypical complex hyperplasia: A meta-analysis and systematic review. Medicine (Baltimore) 96: e8034.

17. Gallos ID, Yap J, Rajkhowa M, Luesley DM, Coomarasamy A, et al. (2012) Regression, relapse, and live birth rates with fertility-sparing therapy for endometrial cancer and atypical complex endometrial hyperplasia: A systematic review and metaanalysis. Am J Obstet Gynecol 207: 266.e1-266.e12.

18. Ramirez PT, Frumovitz M, Bodurka DC, Sun CC, Levenback $C$ (2004) Hormonal therapy for the management of grade 1 endometrial adenocarcinoma: A literature review. Gynecol Oncol 95: 133-138.

19. Ushijima K, Yahata H, Yoshikawa H, Konishi I, Yasugi T, et al. (2007) Multicenter phase II study of fertility-sparing treatment with medroxyprogesterone acetate for endometrial carcinoma and atypical hyperplasia in young women. $J$ Clin Oncol 25: 2798-2803. 